Military Technical College Kobry El-Kobbah Cairo, Egypt

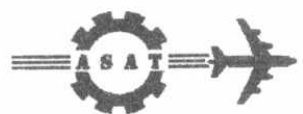

$10^{\text {th }}$ International Conference

On Aerospace Sciences\&

Aviation Technology

\title{
MEASUREMENT AND PRE - PROCESSING OF AUDIOTRY EVOKED RESPONSE DURING ANAESTHESIA
}

\author{
M. Elkfafi ${ }^{*}$, S. Alian*, M.Gadalla*, T.Soliman*.
}

\begin{abstract}
Monitoring of depth of anaesthesia (DOA) is essential and a challenge to anesthetists because it aims to prevent patient's perception of pain, awareness, and recall. However, DOA is very hard to define and not readily measurable. Therefore, a number of monitoring modalities have been considered in an attempt to assess anaesthetic depth and $/$ or the risk of awareness. The mid -latency auditory evoked response (MLAER) has been proposed as the most suitable for monitoring DOA. In this paper, an approach is proposed to enhance the raw auditory evoked response (AER) signal for monitoring the changes in amplitudes and latencies of MLAER waves, which are related to DOA.
\end{abstract}

\section{KEY WORDS}

Depth of Anaesthesia (DOA), Mid Latency Auditory Evoked Response (MLAER), Evoked Potentials (EP), Blood Pressure (BP), Heart Rate (HR), Low Pass Filer (LPF), Electroencephalogram (EEG), Central Nervous System (CNS), Signal to Noise ratio (S/N), High Pass Filter (HPF), Digital Signal Processing (DSP).

* Egyptian Armed Forces. 


\section{I) Introduction}

Both sleep and general anaesthesia are states of unresponsiveness, which vary in depth. Sleep is naturai, where as anaesthesia is an artificial state maintained by the continued presence of chemical agents in brain.

What is anaesthesia? The word "anaesthesia" is derived from the Greek, meaning insensible or without feeling [Collins, 1993]. Arlaesthesia is the art or science of removing sensation of, and reaction to, a surgical procedure. Anaesthesia means loss of all modalities of sensation whether it is the sense of pain, touch, temperature or position. In the use of anaesthetic agents (alone during surgery) there where several complications and side effects, causing death, so the general anaesthesia is applied to decrease the danger of using singie agent during surgery. Any general anaestatic procedure can usually be divided into three components: narcosis (asleep), analgesia (pain relief) and relaxation (muscular relaxation). Therefore modern general anaesthesia (balanced anaesthesia) comprises the triad unconsciousness, muscle relaxation and analgesia [Carrie and Simpson, 1988]. Although general anaesthesia is safer than using single anaesthetic agent, the safety of patient during the surgery still not satisfied.

The Monitor of DOA is complex and dependent on many factors, which vary between the patients and the operating procedures, but it is very important for the anaesthetists to follow the patients during surgery. Also good measuring of DOA provides patient safety, small recovery time, no vomiting and decreases the use of intensive care unites for long time and saves in using drugs.

\section{Depth of Anaesthesia:}

It can be viewed as the position on the scale of pointer as shown in Fig. (1), which reflects the balance between the depressant effects anesthetics on central nervous system and the stimulation by sensory everits such as surgery.

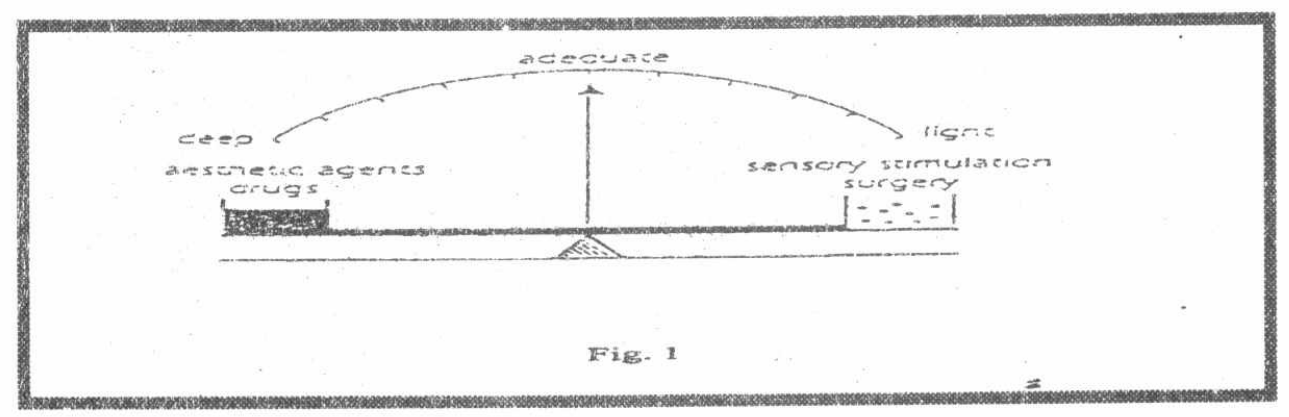

Fig. 1 Definition of DOA 
Therefore, a number of monitoring modalities have been used to measure DOA as shown in table (1) [Thoronton, C., 1991]

Table (1) Monitoring modalities of DOA

\begin{tabular}{|l|} 
1- Mechanical \\
A) Isolated arm \\
B) Esophageal contraction \\
2- Electrical \\
A) Frontal EMG \\
B) EEG \\
1- Direct analysis \\
2- Spectral bands \\
3- Spectral array \\
4- Spectral edge \\
5- Median frequency \\
C) Evoked responses \\
1- Auditory \\
2- Somatosensory \\
3- Visual
\end{tabular}

The value of evoked potentials (EPS) in the clinical assessment of physiological function has been recognized for some considerable time by those specialists neurophysiological interests. More recently, however, the recognition of these techniques as clinically useful tools has spread to anaesthesia. The introduction of muscle relaxants abolishes the traditional signs of anaesthesia, which depends on muscle activity, respiratory changes, movement in response to simulation and muscle tone. Therefore, anaesthetists have used automatic response (change in blood pressure (BP) and heart rate (HR); sweating; lacrimation) as a means of measuring the (DOA). These changes are subject to other drugs used in anaesthesia, such as opioids and anticholinergics, so they are unreliable. The introduction of intravenous anaesthetic agent varies widely between individuals in contrast to the volatile anaesthetic agents. So, the anaesthetic blood level concentration is unreliable as a measure of DOA. Therefore the routine use of intravenous anesthetics combined with neuromuscular blocking agents has created a need for a reliable monitor of DOA. The electroencephalogram (EEG) is an obvious signal to investigate. The EEG, generated from within the central nervous system (CNS), is not affected by neuromuscular blockers, and the raw signal has been known for some time to show graded changes with increasing concentration of anesthetics. However, extensive research has proved disappointing because these changes vary for different agents and no unifying feature has been identified for measuring the DOA in spite of extensive signal processing. 
Attention, therefore, tuned to EPs in the EEG, in the search for a signal which reflects the subjective clinical signs that anaesthetists have used over the years to assess their patients during anaesthesia. The signal should show similar graded changes with anaesthetic concentration for different agents; show appropriate changes with surgical events, and indicates awareness or very light anaesthesia [Lam, 1992]. The EPs, which are changes in electrical potential evoked by auditory, somatosensory or visual stimuli, have two advantages over EEG in the study of anaesthesia:

(a) The EP is an indication of the responsiveness of the CNS whereas the EEG reflects the resting level.

(b) The EPs have anatomical significance.

The Auditory evoked response AER shown in Fig. (2) dose not simply act as a drug concentration monitor but reflects true DOA. Brainstem auditory evoked response (BAER) and late cortical waves of auditory evoked potential cannot be used to measure the anaesthetic depth. The mid latency auditory evoked response (MLAER) waves give dose - related changes with a range of general anaesthetic agents, respond to surgical stimulation, and correlate with cognitive function as well as explicit and implicit memory of events during anaesthesia. Having validated the MLAER as a measure of anaesthetic depth [Thronton and Newton, 1989], we are now proceeding to test it in the clinical context. Its two main drawbacks, averaging time and noise interference, are likely to be overcome using advanced in microprocessor technology.

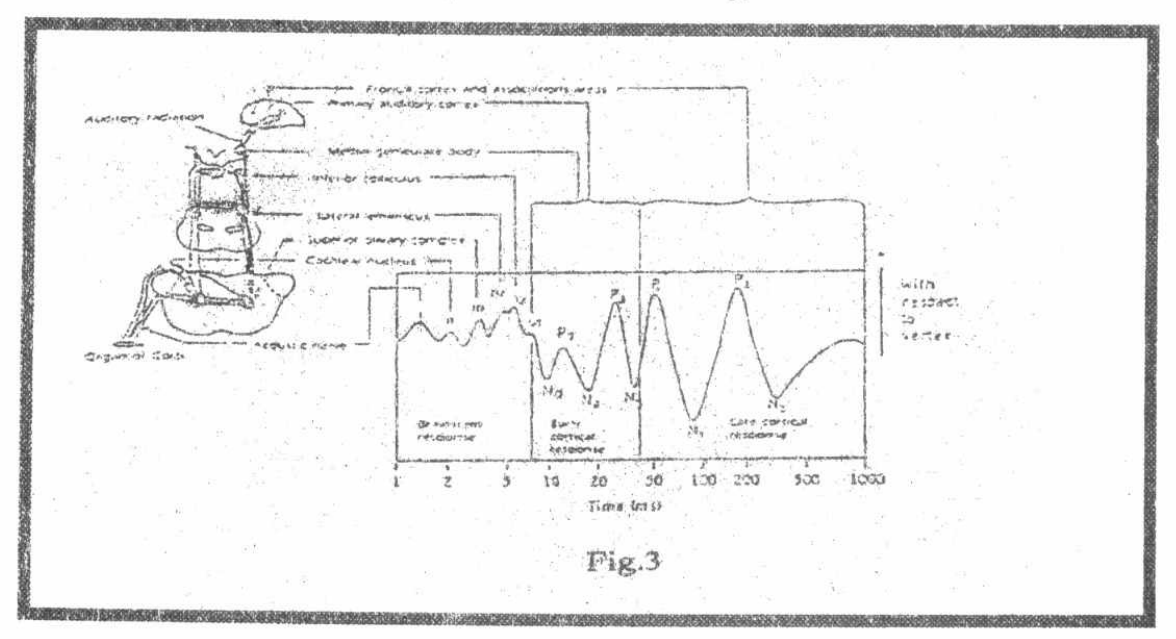

Fig. 2 The generator of AER signal 
The technique of obtaining evoked potentials is shown in Fig. (3), which contains a block diagram of a basic system for recording evoked potentials. The EEG is recorded from scalp electrodes and series of appropriate stimuli (click for auditory, flashes for visual and electric pulses for somatosensory) is applied to a patient. The resulting signals are processed by computer to give an averaged response that is displayed on a VDU screen [Thronton, C., 1991].

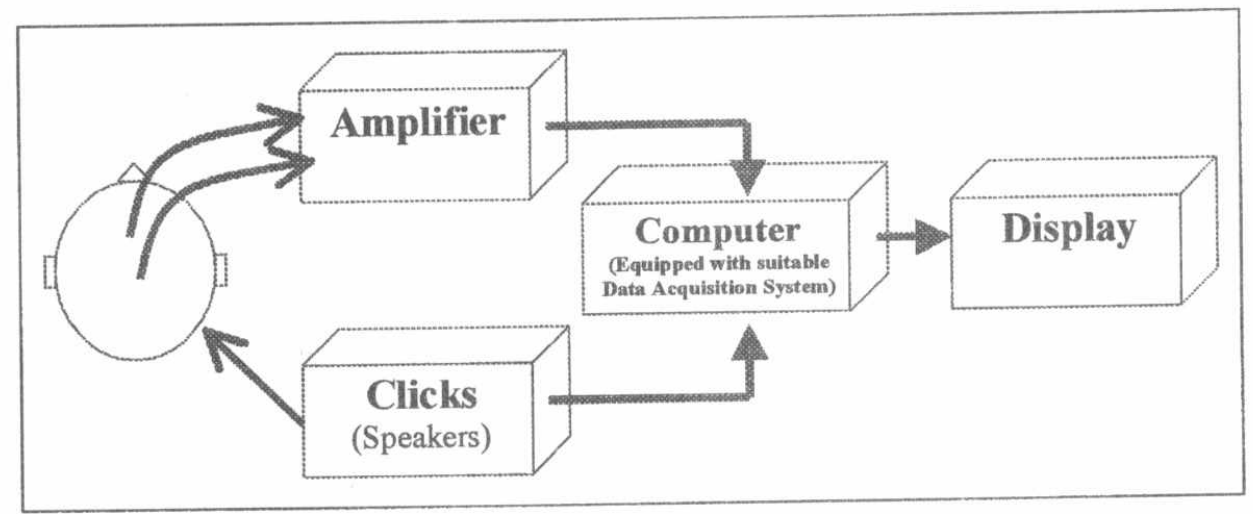

Fig. 3 The technique of obtaining evoked potentials

\section{II) Measurement of AER:}

The acoustic most commonly used for AER measurements is a click applied to both ears simultaneously through earphones designed for audio logical testing. For recording the first $120 \mathrm{~ms}$ of the AER raw data, a $0.4 \mathrm{~ms}$ duration rectangular pulse at repetition frequency of $6.1224 \mathrm{~Hz}$ is commonly used to develop a series of clicks at intensity $75 \mathrm{~dB}$ above the average hearing threshold $(15-20 \mathrm{~dB})$. This stimulus rate is chosen to reject the main supply interference. The reference electrodes $(-v e)$ is placed in the mid forehead and the active electrodes $(+v e)$ is placed in a mastoid site. The preamplifier has self-contained calibration and impedance checking facilities which works in conjunction with software. This is to ensure that the scaling of the EEG signal is known accurately and that there is adequate electrical contact with the patient to minimize the extraneous electrical noise. The electrodes are connected to the patient as described above and their leads are plugged into the preamplifier, which is connected to the computer.

The amplifier and the computer are tuned on and the averaging program runs which will then proceed to estimate the impedance between electrodes connected to the amplifier inputs. Every few seconds the impedance of all electrodes will be adjusted to be less than $5 K \Omega$, then the raw AER data are ready for collection using the averaging program. 
The potential between the two signal electrodes is amplified by a deferential amplifier with gain $\left(10^{5}\right)$ and frequency response of $(1-2000 \mathrm{~Hz})$. Additional analog filtering applied to the signal depending on the desired evoked response. The signal is sampled at rate of $1000 \mathrm{~Hz}$ and filtered with LPF and HPF to obtain the (MLAER). The artifacts are omitted automatically by setting the suitable threshold level of the sweep.

In this work, the conventional averaging technique is applied for averaging (192 sweeps) to enhance the AER signal and making it visible. This value of (192 sweeps) corresponding to a total data acquisition time of just over 30 second, is deemed to small enough to assure that the AER and EEG noise are stationary during the time taking to acquire those 192 sweeps.

After averaging the recording procedure is controlled visually via the computer screen. The recording AER data from patient under anaethesia with propofol during operation are copied to floppy disks for off - line signal processing.

\section{III) Pre - processing of AER}

In this work, the conventional averaging of the small number of sweeps (192sweeps), and filtering technique are applied together to improve S/N of MLAER.

The structure of the system is shown in Fig. (4). The system processes the raw AER signal from off - line AER data that were recorded from patient during anaesthesia with propofol using the technique described above. The conventional averaging technique [Dawson, 1951] is applied to the recorded raw data for small number of sweeps (192 sweeps) to enhance the AER signal. The averaged signal passed through moving average filter, which is suitable for medical processing to smooth it. The length of the moving average filter window for moving average filter is 5 samples.

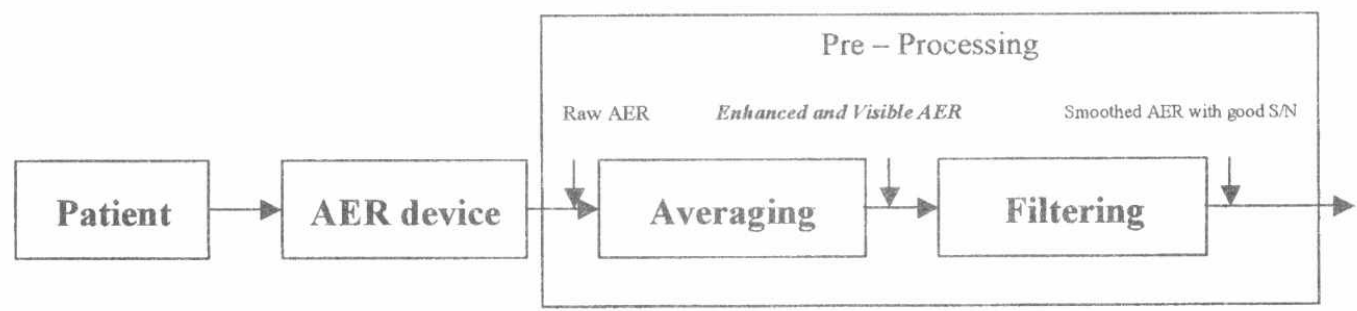

Fig.4 The structure of the system for digital signal processing of AER 


\section{IV) Practical Example:}

In order to test the performance of the DSP system, a simulated MLAER was obtained in the following way.

The case study was based on the data file called AX-7485. The AER data (0-120 ms) of an old patient (64 years) during an operation. These data were recorded during an operation under anaesthesia with propofol at the Royal Hallamshire, Hospital using the AER machine. The raw data was averaged (192 sweeps) using the average program to enhance the MLAER and then applied to moving average filter to smooth the signal and increase the $S / N$.

The raw AER data is shown in fig. (5), the averaged AER signal is shown in Fig. (6), and the output of the DSP system after filtering is shown in Fig. (7). A comparison between the input and the output signals of the DSP system is shown in Fig. (8) to show the increasing in the $S / N$, and the total error is shown in Fig. (9).

\section{V) Conclusion:}

In this paper, the MLAER, as the most suitable signal for monitoring the DOA, has been pre - processed. The pre - processing has been performed via averaging process and filtering. The purpose of this pre - processing is to enhance and increase the S/N of MLAER for preparing it for the next stage, which will be the feature extraction. This stage is complementary work, which is going on.

\section{Vi) References:}

(1) Carrie, L. E. S. and Simpson, P. J., Understanding anesthesia, 2 nd ed. Heinemann Professional Pubs., U. K., pp. 1-6, (1988).

(2) Collins, V.J., Principle of anesthesiology-general and regional anesthesia, $3 \mathrm{rd}$ ed., Vol. 1, Lea \&Febiger, Philadelphia, (1993).

(3) Dawson, G. D., A summation technique for detecting small signals in a large irregular background, J. Phsiol, 115: 2-3, (1951).

(4) Lam,A. M., Do evoked potentials have any value in anaesthesia ?, Anaesthiology Clinics of North America, 10 (3) : 657-681, (1992).

(5) Thronton, C., Evoked potentials in anesthesia, European journal of Anaesthiology, 1991, 8:89-107, (1991).

(6) Thronton, C., and Newton, D. E. F., The auditory evoked response: a measure of depth of anaesthesia, Balliere's Clin. Anaesth., 3: 559-585,(1989). 


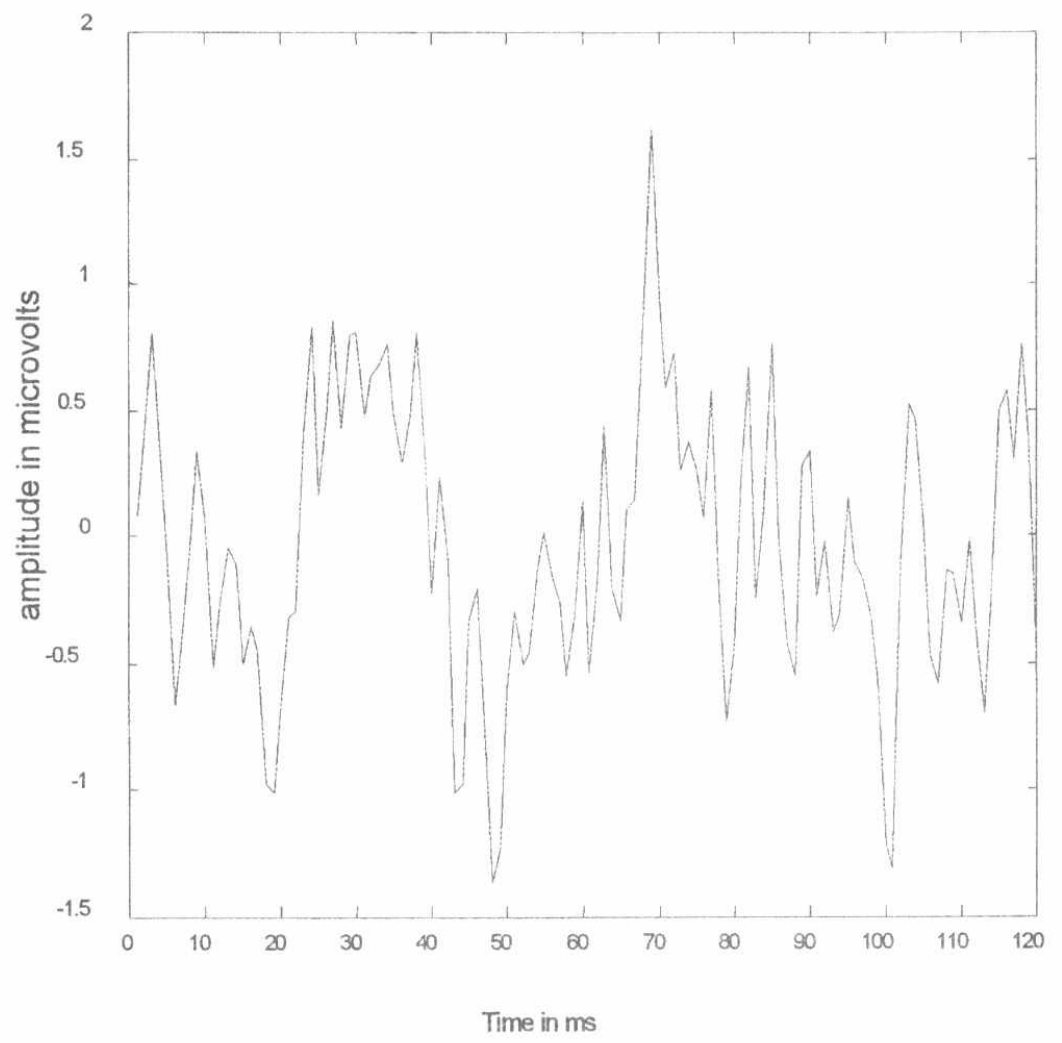

Fig. 5 The raw AER data of patient AX7485 


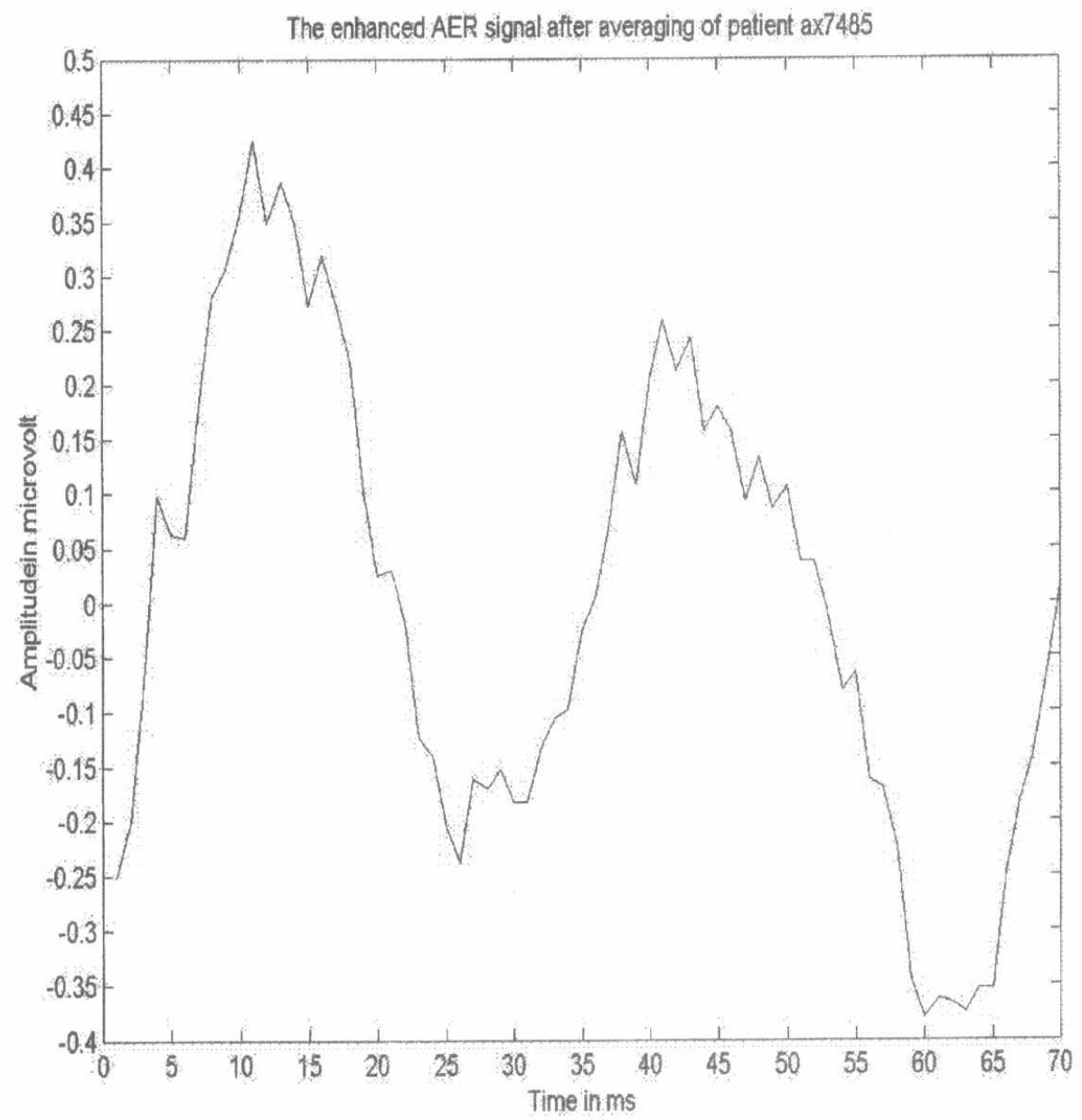

Fig. 6 The enhanced AER signal after averaging of patient AX 7485 


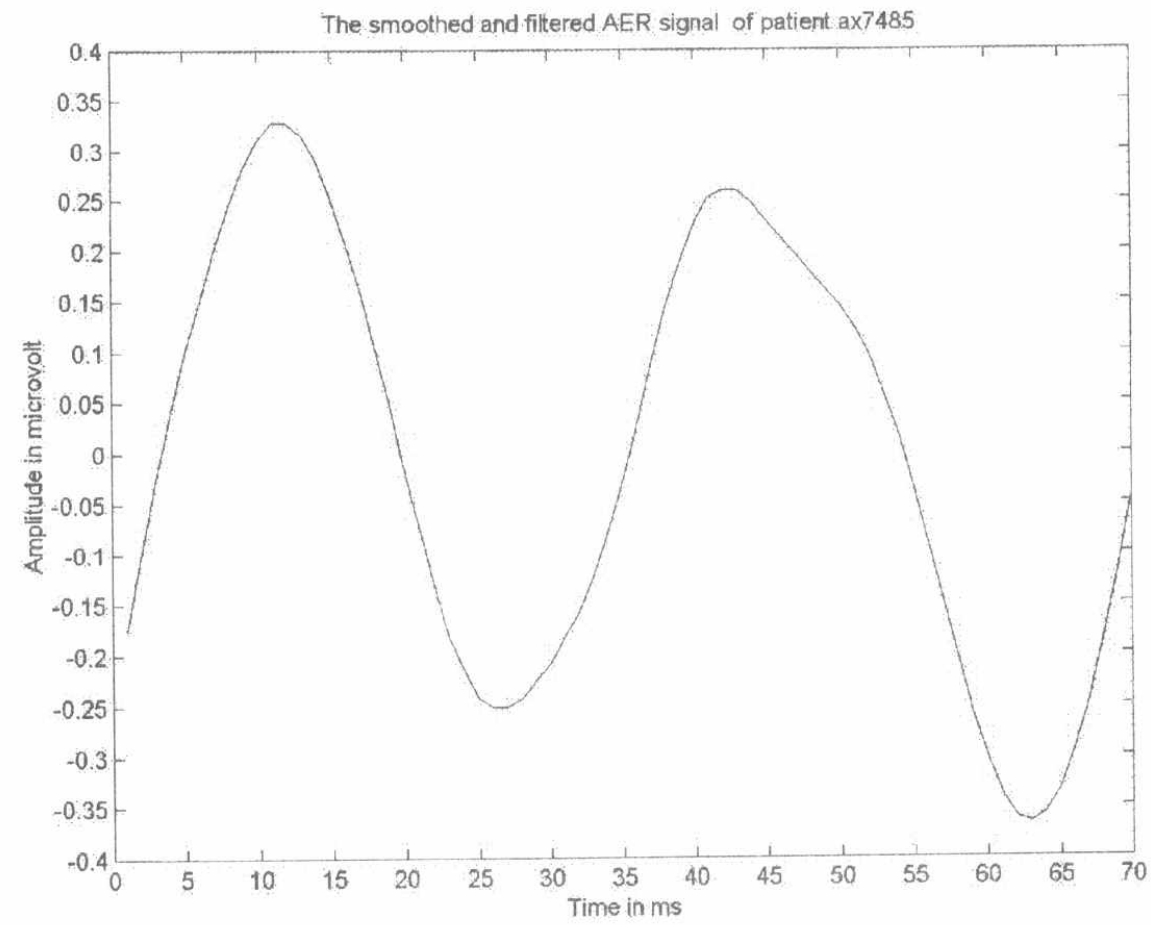

Fig. 7 The smoothed and filtered AER signal of patient AX 7485 


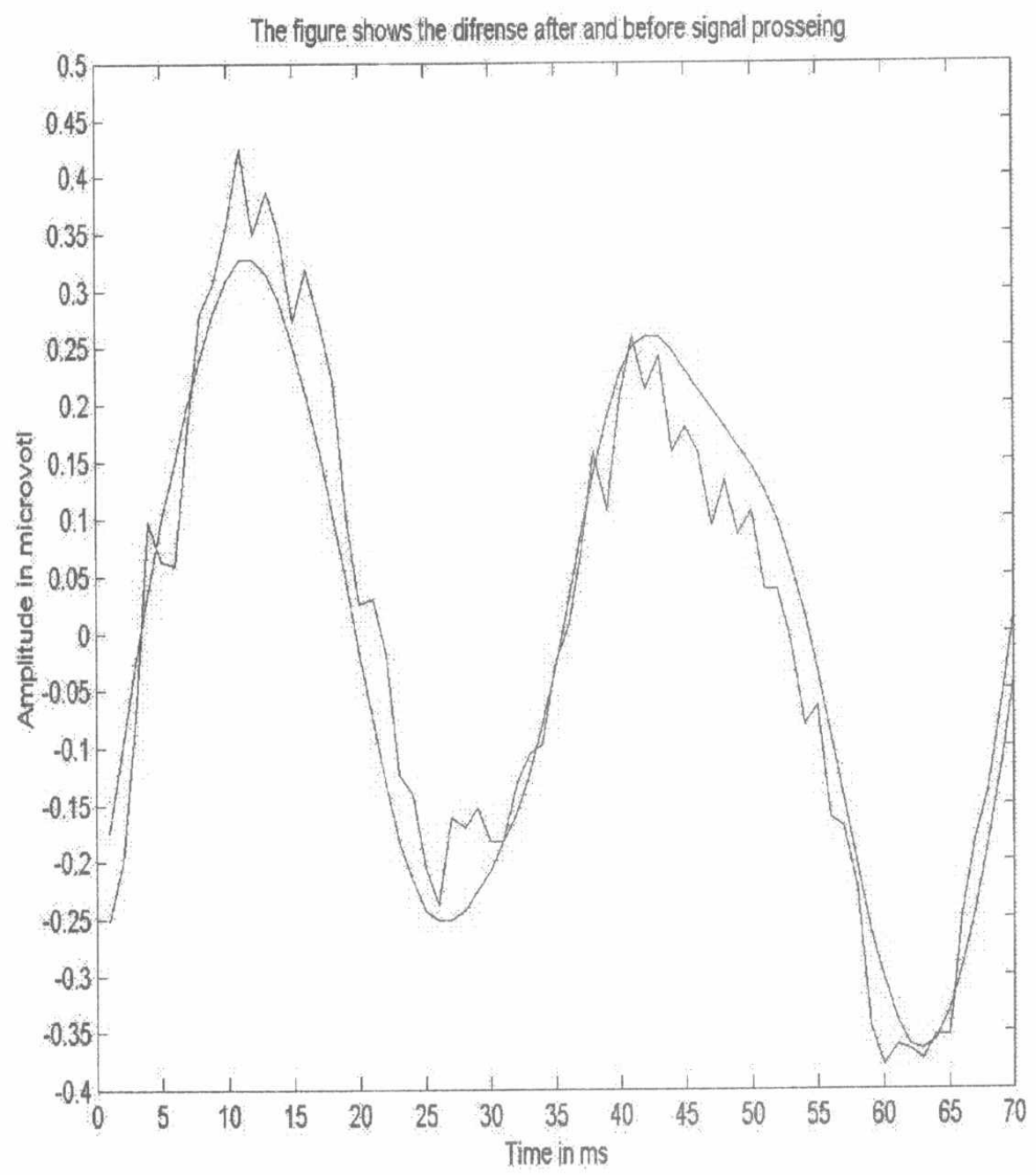

Fig. 8 The comparison between the signals in Fig. (6) and in Fig. (7) 


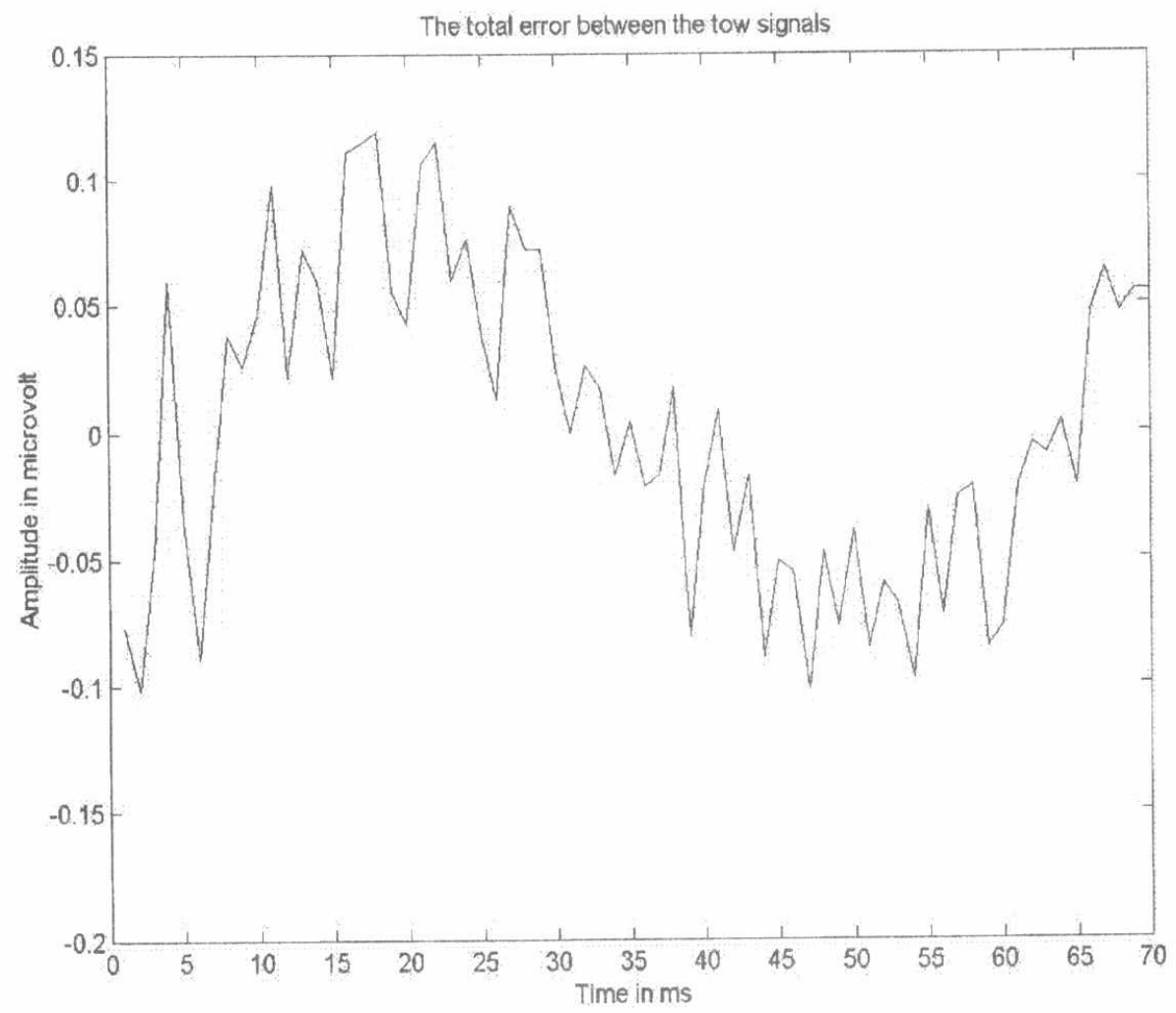

Figure (9) The total error between the signals in Fig. (6) and in Fig. (7) 